\title{
Radial Modes of Neutron Stars with a Quark Core
}

\author{
P.K. Sahu, G.F. Burgio and M. Baldo \\ Istituto Nazionale di Fisica Nucleare, Sezione di Catania \\ Corso Italia 57, I-95129 Catania, Italy
}

\begin{abstract}
We make a first calculation of eigenfrequencies of radial pulsations of neutron stars with quark cores in a general relativistic formalism given by Chandrasekhar. The equations of state (EOS) used to estimate such eigenfrequencies have been derived by taking proper care of the hadron-quark phase transition. The hadronic EOS's have been obtained in the framework of the Brueckner-Hartree-Fock and relativistic mean field theories, whereas the quark EOS has been derived within the MIT bag model. We find that the periods of oscillations of neutron stars with a quark core show a kink, which is associated with the presence of a mixed phase region. Also, oscillation periods show significant differences between ordinary neutron stars and neutron stars with dynamical quark phases.
\end{abstract}

Subject headings: dense matter — equation of state — stars: neutron — stars: oscillations

Nuclear matter at sufficiently high density and temperature is expected to undergo a phase transition to a quark-gluon plasma. The indirect evidences from heavy-ion experiments at CERN (Heinz 2000; Heinz \& Jacobs 2000) and more recently at RHIC (Blaizot 2001) assume to confirm the formation of a quark-gluon plasma. Such a phase transition might occur inside neutron stars, because these are cold and very compact astrophysical objects. It is therefore very interesting to study the effects of possible phase transitions in neutron stars observable like maximum gravitational masses, radii, oscillation frequencies, etc.

In the present letter, we analyze the consequences of a hadron-quark phase transition on the periods of radial oscillations in neutron stars. In fact, more than three decades ago, Cameron (1965) made a suggestion that vibration of neutron stars could excite motions that can have interesting astrophysical implications. There are several investigations of vibrating neutron stars and the simple dimensional analysis suggest that the period of fundamental mode would be the order of milliseconds. More than two decades later, Cutler et al. (1990) concluded that neutron stars of about one solar mass and radius about $10 \mathrm{~km}$ give periods (35) $\mathrm{ms}$, and these turned out to be relatively insensitive to the exact value of central density. 
Also, Datta et al. (1992) tried to calculate the oscillation periods for strange quark stars and they found it to lie in a range of values $0.06-0.3 \mathrm{~ms}$. These values were not substantially different from the ones of conventional neutron stars characterized by the periods $\sim 0.3 \mathrm{~ms}$ for primary mode and $\leq 0.2 \mathrm{~ms}$ for higher modes. Since the neutron stars were assumed to be composed of only hadron matter, the results may be different if a core made of quark matter is present. Of course, the results strictly depend on the construction of the equation of state along with some constraints on the parameters in both hadron and quark phases. Here we are mainly searching for possible signals from the onset of the quark phase, therefore the details of the EOS should not be relevant.

In this letter, we adopt the equations of state for neutron stars with a quark core as developed very recently by Burgio et al. (2001), and then we estimate the period of radial oscillations by using pulsation equations of a nonrotating star in general relativistic formalism given by Chandrasekhar (1964).

The equation of state used here may be divided into three components. The equations of state used in the hadron sector have been derived in the non-relativistic BruecknerHartree-Fock (BHF) and the relativistic mean field (RMF) approaches. In the BHF method (Baldo, 1999), the Brueckner-Bethe-Goldstone formalism has been used with realistic Paris two-body (Lacombe et al., 1980) and Urbana (Carlson et al., 1983; Schiavilla et al., 1986) three-body forces, to ensure the correct reproduction of nuclear matter saturation properties. This procedure has been extended to asymmetric nuclear matter (Baldo et al., 1998; Baldo et al., 2000; Vidaña et al., 2000) including hyperons by implementing hyperon-nucleon potentials that are fitted with existing scattering data. The RMF theory has been derived (Serot \& Walecka, 1986) from the many-body Lagrangian density in the mean field approximation. The parameters are fitted in such a way that it reproduces the correct values of nuclear matter properties at the saturation point (Ghosh et al., 1995; Sahu, 2000). Also hyperons are included in RMF at the mean field level for asymmetric nuclear matter with symmetry energy around $30 \mathrm{MeV}$ at saturation, same as that of BHF theory. The nuclear incompressibility is around $260 \mathrm{MeV}$ for BHF theory and is taken to be same in RMF theory for the sake of compatibility.

The quark matter equation of state has a big uncertainty due to models dependence and its parameters. In the literature, there are models (Schertler et al., 1998) associated with different ad hoc parameters in quark masses and bag constant. We adopted here the simple MIT bag model (Chodos et al., 1974) both with density dependent and independent bag constant. The density dependent bag constant is fixed according to the hypothesis of a constant energy density along the transition line, compatible with the CERN data. Several parametrizations have been considered in recent calculations (Burgio et al., 2001). 
For a Wood-Saxon parametrization of the density dependent bag constant, the onset of quark phase in neutron stars takes place at about two times the saturation density, while for Gaussian like parametrizations the onset occurs at lower density. To be specific we choose the Wood-Saxon parametrization, but the results are expected to be similar for the other parametrizations.

Once the equations of state both in the hadron and the quark sector are well established, we can construct the mixed phase by assuming a first order phase transition. As pointed out by Glendenning (1992), the first order phase transition in neutron stars differs from the one in ordinary matter, because of two conserved charges, i.e. the baryon charge and the global electrical charge. As a consequence, the pressure in the mixed phase varies continuously with the baryon density and is not a constant. The proportion of the hadron and quark component in the mixed phase is then calculated imposing the mechanical and chemical equilibrium, supplemented by the condition of global charge neutrality (Schertler et al., 1998; Burgio et al., 2001). Finally we can construct the total equation of state, which spans from hadron to mixed and quark phases. This is the main ingredient needed to calculate the frequencies of radial modes in neutron stars. It has to be noticed that this equation of state, according to the complete Glendenning' s construction, includes the mixed phase and there is no sharp surface, at a given density (and radius), separating the hadron and the quark phases. This is at variance with the calculations of Haensel et al. (1989) and represents one of the novelty of our calculations. See also Haensel et al. (1990). In agreement with this physical construction, during the star oscillations, the transition from one phase to the other cannot be limited by diffusion. Since the time scale of weak processes are surely much shorter than the period, matter will remain in beta equilibrium and the calculated equation of state is the relevant one for the study of the density oscillations.

The equation for infinitesimal radial pulsations of a nonrotating star was given by Chandrasekhar (1964) and, in general relativity formalism, has the following form:

$$
X \frac{d^{2} \xi}{d r^{2}}+Y \frac{d \xi}{d r}+Z \xi=\sigma^{2} \xi
$$

Here $\xi(\mathrm{r})$ is the Lagrangian fluid displacement and $c \sigma$ is the characteristic eigenfrequency ( $c$ is the speed of light). The quantities $X, Y, Z$ depend on the equilibrium profiles of the pressure $p$ and density $\rho$ of the star and are represented by

$$
X=\frac{-e^{-\lambda} e^{\nu}}{p+\rho c^{2}} \Gamma p
$$




$$
\begin{aligned}
Y & =\frac{-e^{-\lambda} e^{\nu}}{p+\rho c^{2}}\left\{\Gamma p\left(\frac{1}{2} \frac{d \nu}{d r}+\frac{1}{2} \frac{d \lambda}{d r}+\frac{2}{r}\right)\right. \\
& \left.+p \frac{d \Gamma}{d r}+\Gamma \frac{d p}{d r}\right\}, \\
Z & =\frac{e^{-\lambda} e^{\nu}}{p+\rho c^{2}}\left\{\frac{4}{r} \frac{d p}{d r}-\frac{(d p / d r)^{2}}{p+\rho c^{2}}-A\right\} \\
& +\frac{8 \pi G}{c^{4}} e^{\nu} p
\end{aligned}
$$

$\Gamma$ is the adiabatic index defined as

$$
\Gamma=\left(1+\rho c^{2} / p\right) \frac{d p}{d\left(\rho c^{2}\right)}
$$

and

$$
\begin{aligned}
A & =\frac{d \lambda}{d r} \frac{\Gamma p}{r}+\frac{2 p}{r} \frac{d \Gamma}{d r}+\frac{2 \Gamma}{r} \frac{d p}{d r}-\frac{2 \Gamma p}{r^{2}} \\
& -\frac{1}{4} \frac{d \nu}{d r}\left(\frac{d \lambda}{d r} \Gamma p+2 p \frac{d \Gamma}{d r}+2 \Gamma \frac{d p}{d r}-\frac{8 \Gamma p}{r}\right) \\
& -\frac{1}{2} \Gamma p\left(\frac{d \nu}{d r}\right)^{2}-\frac{1}{2} \Gamma p \frac{d^{2} \nu}{d r^{2}} .
\end{aligned}
$$

To solve the pulsations equation (1), the boundary conditions are

$$
\begin{aligned}
\xi(r=0) & =0 \\
\delta p(r=R) & =-\xi \frac{d p}{d r}-\left.\Gamma p \frac{e^{\nu / 2}}{r^{2}} \frac{\partial}{\partial r}\left(r^{2} e^{-\nu / 2} \xi\right)\right|_{r=R} \\
& =0 .
\end{aligned}
$$

It is important to note that $\xi$ is finite, when $p$ vanishes at $r=R$. The pulsations equation (1) is a Sturm - Liouville eigenvalue equation for $\sigma^{2}$, subject to the boundary conditions Eq. (7) and (8). As a consequence the eigenvalues $\sigma^{2}$ are all real and form an infinite discrete sequence $\sigma_{o}^{2}<\sigma_{1}^{2}<\ldots<\sigma_{n}^{2}<\ldots \ldots$, with the corresponding eigenfunction $\xi_{0}(r), \xi_{1}(r), \ldots, \xi_{n}(r)$, where $\xi_{n}(r)$ has $n$ nodes. It immediately follows that if the fundamental radial mode of a star is stable $\left(\sigma_{o}^{2}>0\right)$, then all the radial modes are stable.

We note that Eqs.(2-6) depend on the pressure and density profiles, as well as on the metric functions $\lambda(r), \nu(r)$ of the nonrotating star configuration. Those profiles are obtained by solving the Oppenheimer-Volkoff equations of hydrostatic equilibrium (Misner et al., 1970)

$$
\frac{d p}{d r}=-\frac{G\left(\rho+p / c^{2}\right)\left(m+4 \pi r^{3} p / c^{2}\right)}{r^{2}\left(1-2 G m / r c^{2}\right)},
$$




$$
\begin{aligned}
\frac{d m}{d r} & =4 \pi r^{2} \rho \\
\frac{d \nu}{d r} & =\frac{2 G}{r^{2} c^{2}} \frac{\left(m+4 \pi r^{3} P / c^{2}\right)}{\left(1-2 G m / r c^{2}\right)} \\
\lambda & =-\ln \left(1-2 G m / r c^{2}\right)
\end{aligned}
$$

Eqs. (9) - (12) can be numerically integrated for a given equation of state $\mathrm{p}(\rho)$ and given central density to obtain the radius $R$ and gravitational mass $M=m(R)$ of the star. Therefore the basic input to solve the structure and pulsation equations is the equation of state, $p=p(\rho)$. It has been seen (Burgio et al., 2001) that structure parameters of neutron stars are mainly dominated by the equation of state at high densities, specifically around the core. Since the oscillation features are governed by structure profiles of neutron stars, it is expected to possess marked sensitivity on the high density equation of state as well.

In this calculation, we adopted the equation of states, that have been derived from BHF theory in a non-relativistic limit (Baldo et al., 1998; Baldo et al., 2000) and from RMF theory in a relativistic limit (Ghosh et al., 1995; Sahu, 2000) without quark core. These are denoted by BHF and RMF respectively. Also, we took the equation of states of both BHF and RMF theories with quark core, considering the Wood-Saxon like density dependent parameterization of bag constant (Burgio et al., 2001) in the quark sector. They are represented by $\mathrm{BHF}+\mathrm{MW}$ and $\mathrm{RMF}+\mathrm{MW}$. Another set of equation of states with quark phase were taken with constant bag parameters in quark sector (Datta et al., 1992) along with BHF and RMF theories, where the value of $B=110 \mathrm{MeV} \mathrm{fm}^{-3}$ was chosen in order to ensure the presence of quark matter in the core, for completeness. These are correspondingly labeled by $\mathrm{BHF}+\mathrm{M}$ and $\mathrm{RMF}+\mathrm{M}$. We employed these three sets of equation of states to calculate the oscillation period $P(=2 \pi / c \sigma)$ versus gravitational mass $M$ (in units of solar mass $\left.M_{\odot}\right)$. The results are shown in figures $1-2$.

In both figures, in the upper panel the oscillation periods in seconds are shown versus the total gravitational mass, while in the lower panel the total gravitational mass versus central density is displayed. For the sake of comparison, we show also the results for neutron stars without quark core. If we carefully examine figure 1, we notice that the period of oscillations in $\mathrm{BHF}+\mathrm{MW}$ and $\mathrm{RMF}+\mathrm{MW}$ displays a small kink around the point where the mixed phases start, in the primary as well as in the higher modes. In other words, the period increases and then decreases with respect to the usually decreasing trend observed in BHF and RMF. This happens within a small range of gravitational masses, $0.4<M / M_{\odot}<0.7$, which corresponds to central density, $0.2<\rho_{c} /\left(10^{15} \mathrm{~g} \mathrm{~cm}^{-3}\right)<1.7$, where the mixed phase regions are located. The periods are slightly smaller than $0.4 \mathrm{~ms}$ in $\mathrm{BHF}+\mathrm{MW}$ and RMF+MW models compared with the periods in BHF and RMF models for the fundamental mode and the similar trend 
has been seen in higher modes also. When we compare BHF $+\mathrm{M}$ and $\mathrm{RMF}+\mathrm{M}$ models with BHF and RMF models in figure 2, we notice that there are small kinks in the period of oscillation in the mixed phase regions with gravitational masses $0.5<M / M_{\odot}<1.2$ and corresponding central densities, $0.6<\rho_{c} /\left(10^{15} \mathrm{~g} \mathrm{~cm}^{-3}\right)<1.5$. But these kinks are not as prominent as seen in $\mathrm{BHF}+\mathrm{MW}$ and $\mathrm{RMF}+\mathrm{MW}$ models. This is due to the fact that the transition from hadron to quark phase is smooth in the case of a constant bag parameter. However, the periods of oscillations within these models are comparable with BHF and RMF models for both primary (larger than $0.4 \mathrm{~ms}$ ) and higher modes. Thus significant kinks are observed in BHF $+\mathrm{MW}$ and $\mathrm{RMF}+\mathrm{MW}$ realistic models, because the bag constant is density dependent. The fundamental mode of oscillation periods for neutron stars with and without quark core are found to have the range $0.2-0.6 \mathrm{~ms}$, the only difference being a significant kink in neutron stars which are associated with quark phase. The substantial difference is observed in the fundamental modes of the period of oscillations $(\sim .22 \mathrm{~ms})$ in the neutron stars which are composed of quark cores with density dependence bag parameters $(\mathrm{BHF}+\mathrm{MW}$ and $\mathrm{RMF}+\mathrm{MW})$ from that of normal neutron stars without quark core (BHF and RMF), at the maximum gravitational mass limit (see figure 1). For higher modes the periods are $\leq 0.3 \mathrm{~ms}$ for all the cases. As another interesting point, we notice that all neutron stars with quark core have maximum gravitational masses around $1.5 M_{\odot}$.

In summary, we have presented a calculation of the period of oscillations of neutron stars by using the radial pulsation equations of nonrotating neutron star, as given by Chandrasekhar in general relativity formalism. To solve the radial pulsation equations, one needs a structure profile of nonrotating neutron stars by employing realistic equation of states. The equation of state we used here were derived from the non-relativistic and relativistic formalism with quark phase at higher densities. Since the quark matter is not well established, we explored the parameters of quark matter compatible with the heavy-ion experiments at the point of possible formation of quark-gluon plasma. Then the equation of state were constructed by using Glendenning condition for mechanical and chemical equilibrium as a function of baryon and electron density at the mixed phase, comprising with hadron, mixed and quark phases. The main conclusion of our work is that the period of oscillations shows some significant kink against gravitational mass, if one uses a realistic equation of state associated with density dependent bag constant. These type of kinks are not present in conventional neutron stars, constituted by only hadrons. These kinks can be considered a distinct signature of the quark matter onset in neutron stars.

\section{REFERENCES}

Baldo, M., Burgio, G. F., \& Schulze, H.-J. 1998, Phys.Rev.C, 58, 3688 
Baldo, M. 1999, in Nuclear Methods and The Nuclear Equation of State, ed. M. Baldo (Singapore: World Scientific), 1

Baldo, M., Burgio, G. F., \& Schulze, H.-J. 2000, Phys.Rev.C, 61, 055801

Blaizot, J. P. 2001, preprint(nucl-th/0107025)

Burgio, G. F., Baldo, M., Sahu, P. K., Santra, A. B., \& Schulze H. -J. 2001, Phys.Lett.B, submitted

Cameron, A. G. W. 1965, Nature, 205, 787

Carlson, J., Pandharipande, V. R., \& Wiringa, R. B. 1983, Nucl.Phys.A, 401, 59

Chandrasekhar, S. 1964, ApJ, 140, 417

Chodos, A., Jaffe, R. L., Johnson K., Thorn, C. B., \& Weisskopf, V. F. 1974, Phys.Rev.D, 9,3471

Cutler, C., Lindblom, L. \& Splinter, R. J. 1990, ApJ, 363, 603

Datta, B., Sahu, P. K., Anand, J. D., \& Goyal, A. 1992, Phys.Lett.B, 283, 313

Datta, B., Hasan, S. S., Sahu, P. K., \& Prasanna, A. R. 1998, Int.Jour.Mod.Phys.D, 7, 49

Ghosh, S. K., Phatak, S. C. \& Sahu, P. K. 1995, Z. Phys.A, 352, 457

Glendenning, N. K. 1992, Phys.Rev.D, 46, 1274

Haensel, P., Zdunik, J.L. \& Schaeffer, R. 1989, A\&A 217, 137

Haensel, P., Denissov, A. \& Popov, S. 1990, A\&A 240, 78

Heinz, U., \& Jacobs, M. 2000, preprint (nucl-th/0002042)

Heinz, U. 2000, preprint (hep-ph/0009170)

Lacombe, M., Loiseau, B., Richard, J. M., Vinh Mau, R., Côté, J., Pirès, P., \& de Tourreil, R. 1980, Phys.Rev.C, 21, 861

Misner, C. W., Thorne, K. S., \& Wheeler, J. A. 1970, Gravitation (San Francisco; W. H. Freeman ed.)

Sahu, P. K. 2000, Phys.Rev.C, 62, 045801

Schertler, K., Greiner, C., Sahu, P. K., \& Thoma M. H. 1998, Nucl.Phys.A, 637, 451 
Schiavilla R., Pandharipande, V. R., \& Wiringa, R. B. 1986, Nucl.Phys.A, 449, 219

Serot, B. D., \& Walecka, J. D. 1986, Adv.Nucl.Phys., 16, 1

Vidaña, I., Polls, A., Ramos, A., Engvik, L., \& Hjorth-Jensen, M. 2000, Phys.Rev.C, 62, 035801

This preprint was prepared with the AAS IATEX macros v5.0. 

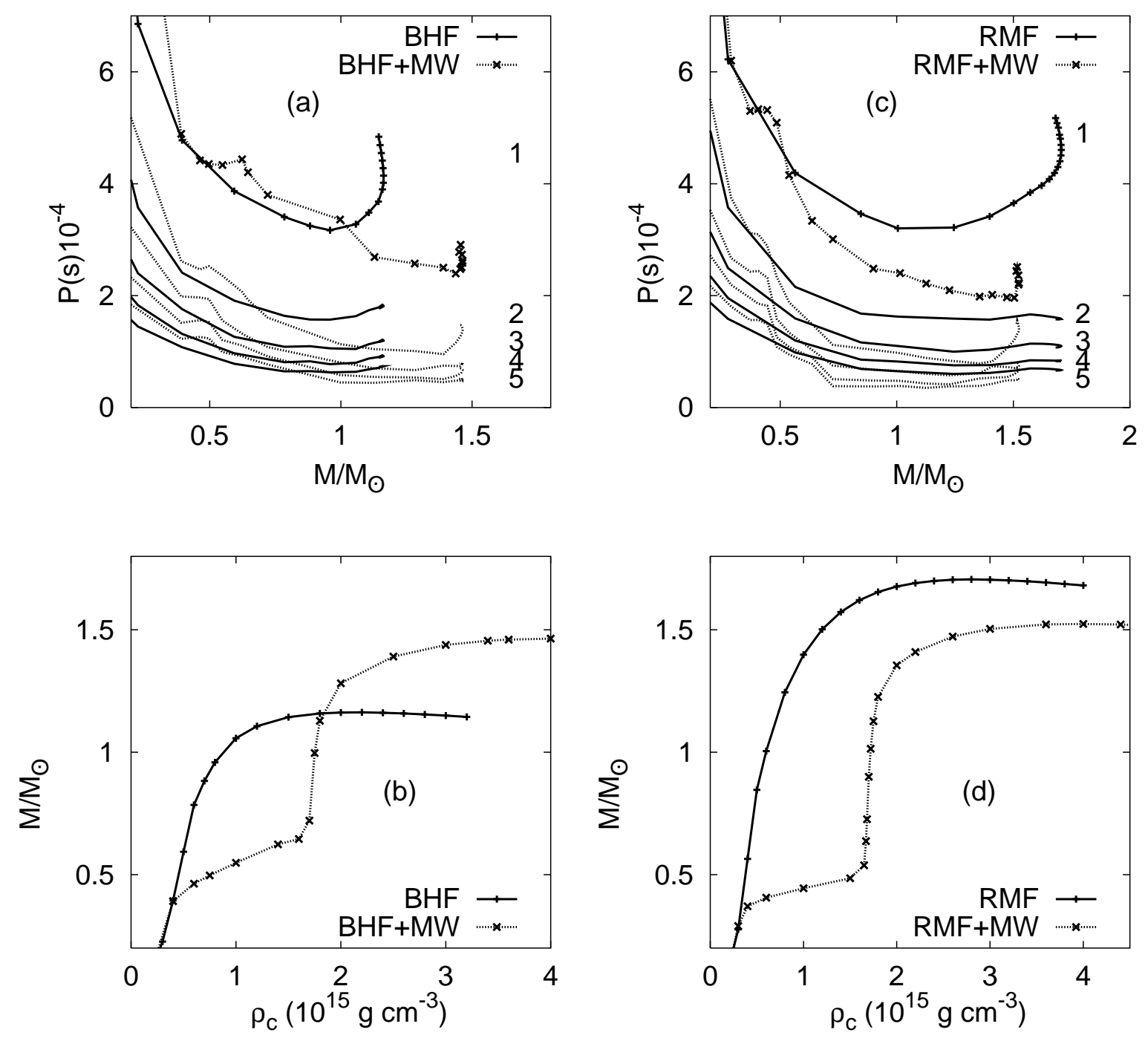

Fig. 1.- In the upper panels the oscillation period in seconds is displayed vs. the gravitational mass in units of the solar mass, whereas in lower panels the gravitational mass is shown vs. the central density. Panels (a) and (b) show results for BHF calculation for purely hadronic (solid line) and mixed hadron-quark matter (dotted line). The bag constant is assumed to be density dependent. Labels 1,2, etc. indicate the higher modes. Panels (c) and (d) correspond to RMF calculations. 

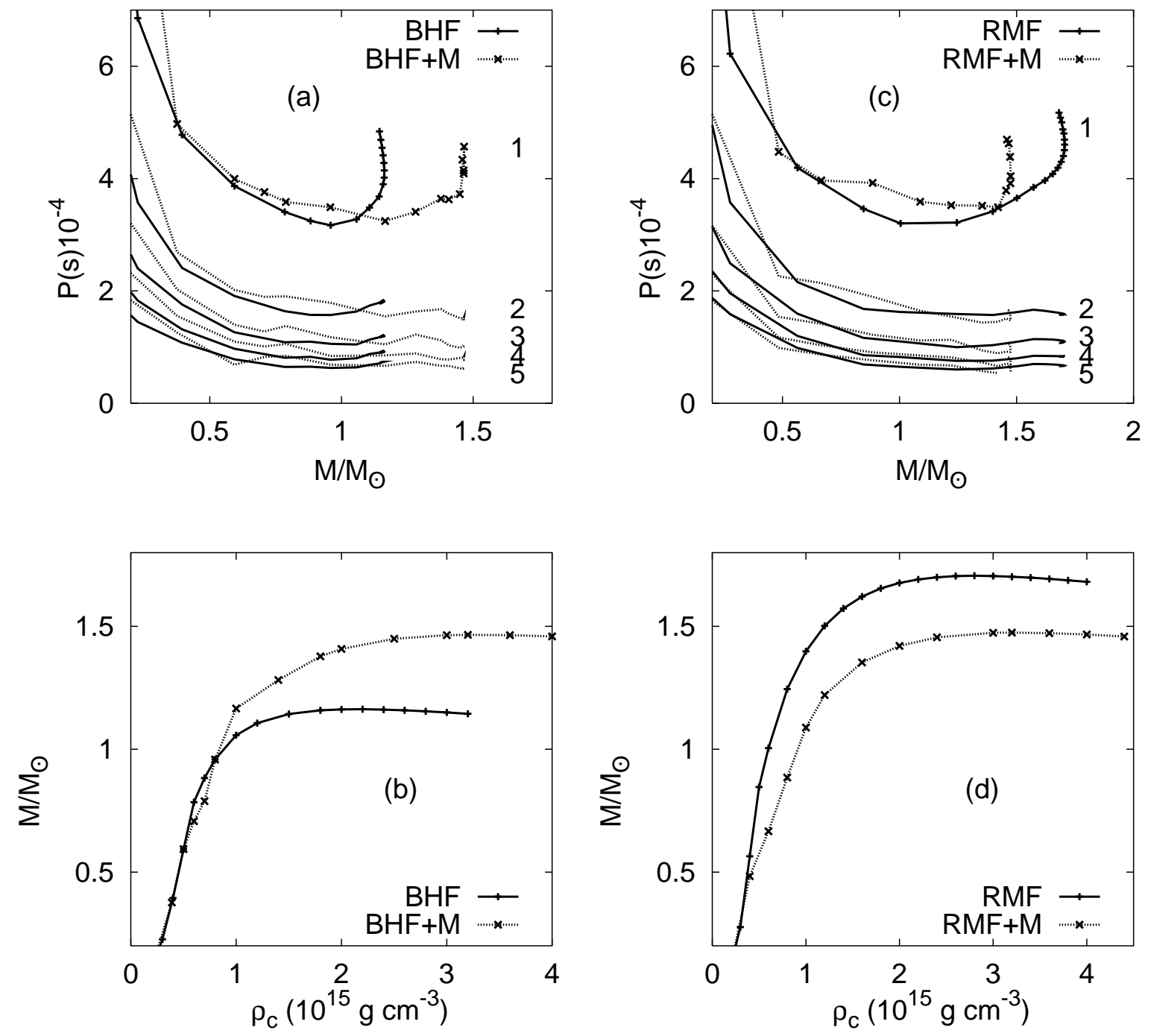

Fig. 2.- Same as figure 1, but for density independent bag constant. See text for details. 